MATHEMATICS OF COMPUTATION

Volume 76, Number 259, July 2007, Pages 1275-1290

S 0025-5718(07)01831-5

Article electronically published on February 16, 2007

\title{
FOURIER-PADÉ APPROXIMATIONS AND FILTERING FOR SPECTRAL SIMULATIONS OF AN INCOMPRESSIBLE BOUSSINESQ CONVECTION PROBLEM
}

\author{
M. S. MIN, S. M. KABER, AND W. S. DON
}

\begin{abstract}
In this paper, we present rational approximations based on Fourier series representation. For periodic piecewise analytic functions, the well-known Gibbs phenomenon hampers the convergence of the standard Fourier method. Here, for a given set of the Fourier coefficients from a periodic piecewise analytic function, we define Fourier-Padé-Galerkin and Fourier-Padé collocation methods by expressing the coefficients for the rational approximations using the Fourier data. We show that those methods converge exponentially in the smooth region and successfully reduce the Gibbs oscillations as the degrees of the denominators and the numerators of the Padé approximants increase.

Numerical results are demonstrated in several examples. The collocation method is applied as a postprocessing step to the standard pseudospectral simulations for the one-dimensional inviscid Burgers' equation and the twodimensional incompressible inviscid Boussinesq convection flow.
\end{abstract}

\section{INTRODUCTION}

In this paper, we discuss a rapidly converging approximation to a finite Fourier series by defining a rational function whose denominator and numerator are represented as a finite Fourier sum. The underlying principle of rational approximation was proposed by H. Padé in 1892 [4, 13]. With the advent of computer in the 1950s, Padé rational approximations have become a popular computational method for representing functions, especially rapidly converging functions. They are generally more efficient than polynomial approximations, with a reduced number of operations at the same accuracy [5, 6, 7, 8, 16.

The main objective in this paper is to present simple algorithms for computing Fourier-Padé rational approximations for discontinuous functions. The algorithms are applied as a filter to accelerate the convergence of the oscillatory pseudospectral solutions of nonlinear partial differential equations, in particular the inviscid Burgers' equation and incompressible Boussinesq convection flow in the absence of viscosity.

Fourier methods are powerful numerical tools for approximating periodic analytic functions with spectral convergence. However, with periodic piecewise analytic

Received by the editor June 3, 2003 and, in revised form, July 7, 2004.

2000 Mathematics Subject Classification. Primary 41A20, 41A21, 41A25, 65T10, 65T20.

Key words and phrases. Rational approximation, Gibbs phenomenon, Fourier-Padé-Galerkin method, Fourier-Padé collocation, postprocessing.

This research was supported by Grant AFOSR F49620-02-1-0113. 
functions, the well-known difficulty of the Fourier methods is the slow rate of convergence $O\left(\frac{1}{N}\right)$ globally and the oscillations of $O(1)$ near singularity, the so-called Gibbs phenomenon 9, 10, 11.

In order to overcome the oscillatory behavior of the Fourier methods for discontinuous problems, many numerical techniques have been developed. For a given finite set of Fourier data, the Gegenbauer reconstruction techniques [12 remove the oscillations completely, with spectral convergence up to the discontinuity as the degrees of Gegenbauer polynomial expansion increase, when reasonably accurate information for the location of discontinuities is provided.

With no knowledge of the singularity, however, Padé reconstruction successfully recovers a nonoscillatory solution with a reduced overshoot at the singularity. The reason is that the possible existence of poles of some order for the denominator of the Padé approximant allows a better approximation to those functions exhibiting singular behavior, such as large gradient and discontinuity.

In [8], Geer presents a method for implementing the rational trigonometric approximations for even or odd $2 \pi$-periodic piecewise smooth functions and the application to the solution of an initial boundary value problem for a simple heat equation. In his work, Fourier-Padé approximants are defined in a nonlinear way such that the relation between the coefficients of the rational approximations and the Fourier coefficients involves a necessary procedure of calculating the integration of rational functions, which makes the numerical scheme relatively complicated. Moreover, Geer studies only the case for even and odd functions, not the general case.

In [7, Fourier expansion is treated as a Laurent expansion. Using a FourierPadé rational approach, the authors obtain the spectral convergence up to the discontinuity by subtracting the jump from the Fourier data. This approach has the disadvantage of requiring advance knowledge of the singularity.

Here we present two Fourier-Padé methods for the general case of piecewise analytic functions, with no advance knowledge of the singularity. We demonstrate simple ways of implementing Fourier-Padé-Galerkin and Fourier-Padé collocation methods, and we apply these methods to simulate the solutions of nonlinear partial differential equations. For hyperbolic partial differential equations such as Burgers' equation, an initially smooth function can evolve into shock in time for the inviscid case and into a large gradient for the viscous case. Therefore, the standard spectral simulations will exhibit the Gibbs phenomenon and reduce the accuracy of the numerical solutions in time. From accurate Fourier data computed by the Fourier method, we apply the Fourier-Padé reconstruction as a postprocessing step. After postprocessing, the computational results show successful reduction of the nonphysical oscillations in the standard spectral solutions of the one-dimensional inviscid Burgers' equation and the two-dimensional inviscid Boussinesq equations. Further study is needed to find the optimal relation between the degrees of the polynomial of the Padé approximants and the number of the Fourier coefficients.

This paper is organized as follows. In Section 2, the Fourier-Padé-Galerkin method is discussed. The Fourier-Padé-Galerkin coefficients of the denominator are explicitly represented as a linear combination of the given Fourier coefficients. Numerical computations with this method are carried out with the sawtooth function. Section 3 introduces a new method using collocation points. Explicit formulations for the Fourier-Padé collocation coefficients are presented in a manner similar 
to that used to present the Fourier-Padé-Galerkin method. The sawtooth function is again used for the numerical simulations. Section 4 demonstrates the success of the Fourier-Padé collocation method in mitigating the oscillations near the sharp gradient for the spectral solutions of the one-dimensional inviscid Burgers' equation and the two-dimensional incompressible inviscid Boussinesq convection flow. Section 5 summarizes our results.

\section{Fourier-PAdÉ-Galerkin APPROXIMATION}

Let $\mathcal{S}_{N}$ be the space of trigonometric polynomials of degree $N$ defined as

$$
\mathcal{S}_{N}=\operatorname{span}\left\{e^{i n x} \mid-N \leq n \leq N\right\} .
$$

Consider a piecewise analytic $2 \pi$-periodic function $u(x)$ in $[-\pi, \pi]$. We define the Fourier-Padé-Galerkin approximation of $u$ by

$$
R_{K, M}=\frac{P_{K}}{Q_{M}},
$$

where $P_{K} \in \mathcal{S}_{K}$ and $Q_{M} \in \mathcal{S}_{M}$, such that

$$
\left(Q_{M} u-P_{K}, v\right)=0 \text { for all } v \in \mathcal{S}_{K+M},
$$

with the inner product defined by

$$
(u, v)=\frac{1}{2 \pi} \int_{-\pi}^{\pi} u(x) \overline{v(x)} d x .
$$

Let us denote

$$
P_{K}=\sum_{k=-K}^{K} b_{k} e^{i k x} \text { and } Q_{M}=\sum_{m=-M}^{M} c_{m} e^{i m x} .
$$

The Fourier series representation of $u$ is expressed by

$$
u(x)=\sum_{n=-\infty}^{\infty} \hat{u}_{n} e^{i n x},
$$

where

$$
\hat{u}_{n}=\frac{1}{2 \pi} \int_{-\pi}^{\pi} u(x) e^{-i n x} d x .
$$

Then, substituting $P_{K}, Q_{M}$ and $v=e^{i n x}$ for $-(K+M) \leq n \leq K+M$ to (2.3), we obtain the following equations:

$$
b_{k}=\sum_{m=-M}^{M} c_{m} \hat{u}_{k-m} \quad \text { for }|k| \leq K
$$

and

$$
\sum_{m=-M}^{M} c_{m} \hat{u}_{n-m}=0 \quad \text { for } K<|n| \leq K+M .
$$


The system of equation (2.9) can be written in a matrix form, $\mathbf{A c}=0$, where $\mathbf{c}=\left[c_{M}, \ldots, c_{-M}\right]^{T}$ and $2 M \times(2 M+1)$ matrix $\mathbf{A}$ defined by

$$
\mathbf{A}=\left[\begin{array}{ccccc}
\hat{u}_{-(K+2 M)} & \cdots & \hat{u}_{-(K+M)} & \cdots & \hat{u}_{-K} \\
\hat{u}_{-(K+2 M-1)} & \cdots & \hat{u}_{-(K+M-1)} & \cdots & \hat{u}_{-(K-1)} \\
\vdots & & & & \vdots \\
\hat{u}_{-(K+M+1)} & \cdots & \hat{u}_{-(K+1)} & \cdots & \hat{u}_{-(K-M+1)} \\
\hat{u}_{K-M+1} & \cdots & \hat{u}_{K+1} & \cdots & \hat{u}_{K+M+1} \\
\vdots & & & & \vdots \\
\hat{u}_{K-1} & \cdots & \hat{u}_{K+M-1} & \cdots & \hat{u}_{K+2 M-1} \\
\hat{u}_{K} & \cdots & \hat{u}_{K+M} & \cdots & \hat{u}_{K+2 M}
\end{array}\right] .
$$

We solve this linear system numerically using the MATLAB subroutine Null(A), which gives the basis of the kernel of the $2 M \times(2 M+1)$ matrix $A$. Once we obtain a set of the coefficients $\left\{c_{m}\right\}_{m=-M}^{M}$, the coefficients $\left\{b_{k}\right\}_{k=-K}^{K}$ can be computed from (2.8). Note that the continuous Fourier coefficients involved in computing $b_{k}$ and $c_{m}$ are

$$
\left\{\hat{u}_{n}\right\}_{n=-(K+2 M)}^{K+2 M},
$$

and we denote $N=K+2 M$.

Example 1. We demonstrate the numerical results of the Fourier-Padé-Galerkin approximation for a sawtooth function

$$
u(x)= \begin{cases}x+\pi & \text { for } x \in[-\pi, 0), \\ x-\pi & \text { for } x \in[0, \pi],\end{cases}
$$

for which the continuous Fourier coefficients are $\hat{u}_{0}=0$ and $\hat{u}_{n}=-i / n$ for $n=$ $\pm 1, \pm 2, \ldots$

The left figure in Figure 2.1 illustrates the Gibbs phenomenon when the standard Fourier-Galerkin approximation is applied to the sawtooth function for the number of Fourier modes $N=8,16,32$. The right figure in Figure 2.1]shows the reconstructed sawtooth function using the Fourier-Padé-Galerkin approximant (solid line). The Gibbs oscillation is clearly being reduced near the discontinuity.
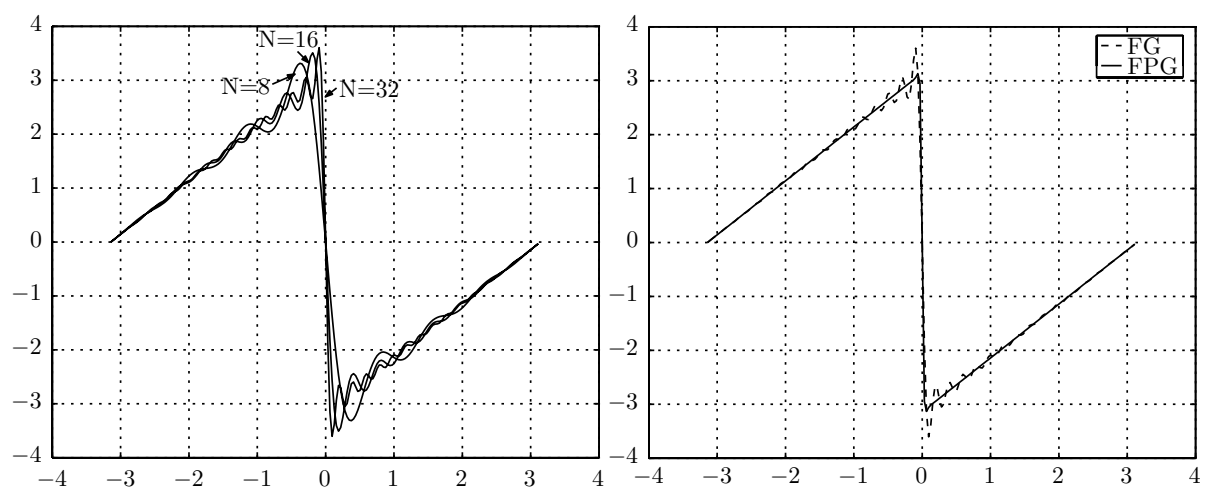

Figure 2.1. Gibbs oscillations by Fourier-Galerkin (FG) approximation (left) and Fourier-Padé-Galerkin (FPG) reconstruction (right) for the sawtooth function. 

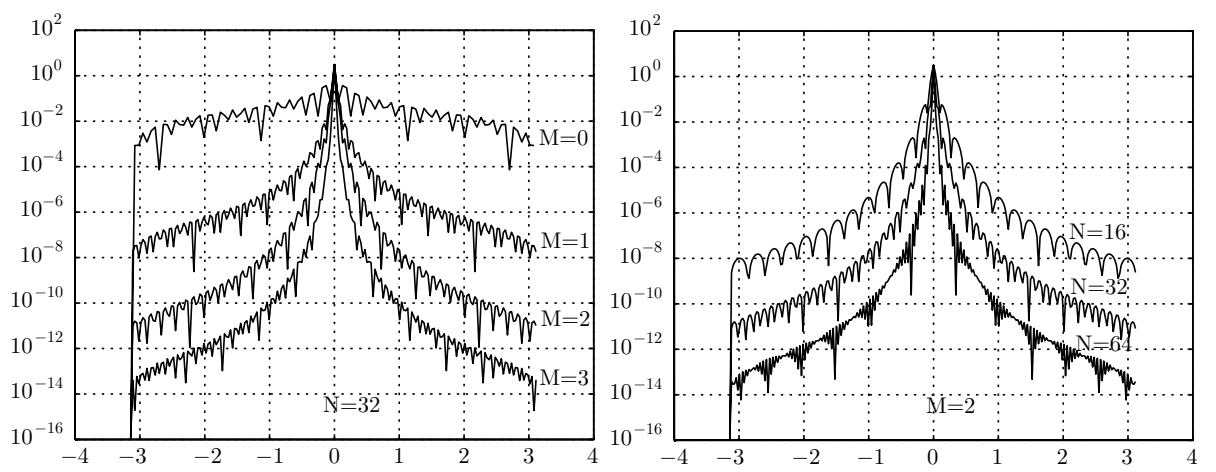

Figure 2.2. Convergence of the pointwise errors after the Fourier-Padé-Galerkin reconstruction for the sawtooth function with a fixed $N$ (left) and a fixed $M$ (right), respectively.

The pointwise errors are shown in Figure 2.2, In the left figure, for a given fixed $N=32$, the degree $M$ of the denominator of the Padé approximant is increased. For $M=0$, it is exactly the standard Fourier-Galerkin method. The pointwise errors decay like $O\left(\frac{1}{N}\right)$ away from the discontinuity, and $O(1)$ accuracy is observed clearly at the location of the jump. By increasing the degree $M$ of the denominator, the errors decay exponentially away from the discontinuity as $M$ increases. For a fixed $M=2$, the rate of convergence of the pointwise error away from the discontinuity is only linear as $N$ increases.

\section{Fourier-Padé collocation approximation}

Let $\mathcal{I}_{N}$ be the space of the trigonometric polynomial of degree $N$, defined as

$$
\mathcal{I}_{N}=\operatorname{span}\left\{e^{i n x} \mid-N \leq n \leq N-1\right\} .
$$

Consider a set of even number of grid points

$$
x_{j}=-\pi+\frac{\pi j}{N}, \quad j=0, \ldots, 2 N-1 .
$$

We define the Fourier-Padé collocation approximation of $u$ by $R_{K, M}^{c}=\frac{P_{K}^{c}}{Q_{M}^{c}}$, where $P_{K}^{c} \in \mathcal{I}_{K}$ and $Q_{M}^{c} \in \mathcal{I}_{M}(K+2 M=N)$, such that

$$
\left(Q_{M}^{c} u-P_{K}^{c}, v\right)_{N}=0 \text { for all } v \in \mathcal{I}_{K+M},
$$

where the discrete inner product is defined by

$$
(u, v)_{N}=\frac{1}{2 N} \sum_{j=0}^{2 N-1} u\left(x_{j}\right) \overline{v\left(x_{j}\right)} .
$$

Denote

$$
P_{K}^{c}=\sum_{k=-K}^{K-1} \tilde{b}_{k} e^{i k x} \text { and } Q_{M}^{c}=\sum_{m=-M}^{M} \tilde{c}_{m} e^{i m x} .
$$


Then, substituting $P_{K}^{c}, Q_{M}^{c}$ and $v=e^{i n x}$ for $-(K+2 M) \leq n \leq K-1+2 M$ to (3.3), we obtain the following equations: for $-K \leq k \leq K-1$,

$$
\tilde{b}_{k}=\sum_{m=-M}^{M} \tilde{c}_{m} \tilde{u}_{k-m}
$$

and, for $-(K+M) \leq n<-K$ and $K-1<n \leq K-1+M$,

$$
\sum_{m=-M}^{M} c_{m} \tilde{u}_{n-m}=0
$$

where

$$
\tilde{u}_{n}=\frac{1}{2 N} \sum_{j=0}^{2 N-1} u\left(x_{j}\right) e^{-i n x_{j}} .
$$

Following a procedure similar to that in Section 2, we have a linear system $\tilde{A} \tilde{\mathbf{c}}=0$ to solve $\tilde{\mathbf{c}}$, where $\tilde{\mathbf{c}}=\left[\tilde{c}_{M}, \ldots, \tilde{c}_{-M}\right]^{T}$ and

$$
\tilde{A}=\left[\begin{array}{ccccc}
\tilde{u}_{-(K+2 M)} & \cdots & \tilde{u}_{-(K+M)} & \cdots & \tilde{u}_{-K} \\
\tilde{u}_{-(K+2 M-1)} & \cdots & \tilde{u}_{-(K+M-1)} & \cdots & \tilde{u}_{-(K-1)} \\
\vdots & & & & \vdots \\
\tilde{u}_{-(K+M+1)} & \cdots & \tilde{u}_{-(K+1)} & \cdots & \tilde{u}_{-(K-M+1)} \\
\tilde{u}_{K-M+1} & \cdots & \tilde{u}_{K+1} & \cdots & \tilde{u}_{K+M+1} \\
\vdots & & & & \vdots \\
\tilde{u}_{K-1} & \cdots & \tilde{u}_{K+M-1} & \cdots & \tilde{u}_{K+2 M-1}
\end{array}\right] .
$$

We solve this linear system numerically using the MATLAB subroutine $\operatorname{Null}(\tilde{A})$, which gives the basis of the kernel of the $2 M \times(2 M+1)$ matrix $\tilde{A}$. Once we obtain a set of the coefficients $\left\{\tilde{c}_{m}\right\}_{m=-M}^{M}$, the coefficients $\left\{\tilde{b}_{k}\right\}_{k=-K}^{K-1}$ can be computed from (3.6).

Note that the discrete Fourier coefficients involved in computing $\tilde{b}_{k}$ and $\tilde{c}_{m}$ are

$$
\left\{\tilde{u}_{n}\right\}_{n=-(K+2 M)}^{K+2 M-1} .
$$

Remark 3.1. The Fourier-Padé collocation approach does not interpolate the function $u(x)$ at the collocation points $x_{j}=-\pi+\frac{\pi j}{N}$. Since from (3.6)

$$
\begin{aligned}
\tilde{b}_{k} & =\sum_{m=-M}^{M} \tilde{c}_{m}\left(\frac{1}{2 N} \sum_{j=0}^{2 N-1} u\left(x_{j}\right) e^{-i(k-m) x_{j}}\right) \\
& =\frac{1}{2 N} \sum_{j=0}^{2 N-1} u\left(x_{j}\right) Q_{M}^{c}\left(x_{j}\right) e^{-i k x_{j}},
\end{aligned}
$$

we have

$$
P_{K}^{c}(x)=\sum_{j=0}^{2 N-1} u\left(x_{j}\right) Q_{M}^{c}\left(x_{j}\right)\left(\frac{1}{2 N} \sum_{k=-K}^{K-1} e^{i k\left(x-x_{j}\right)}\right) .
$$



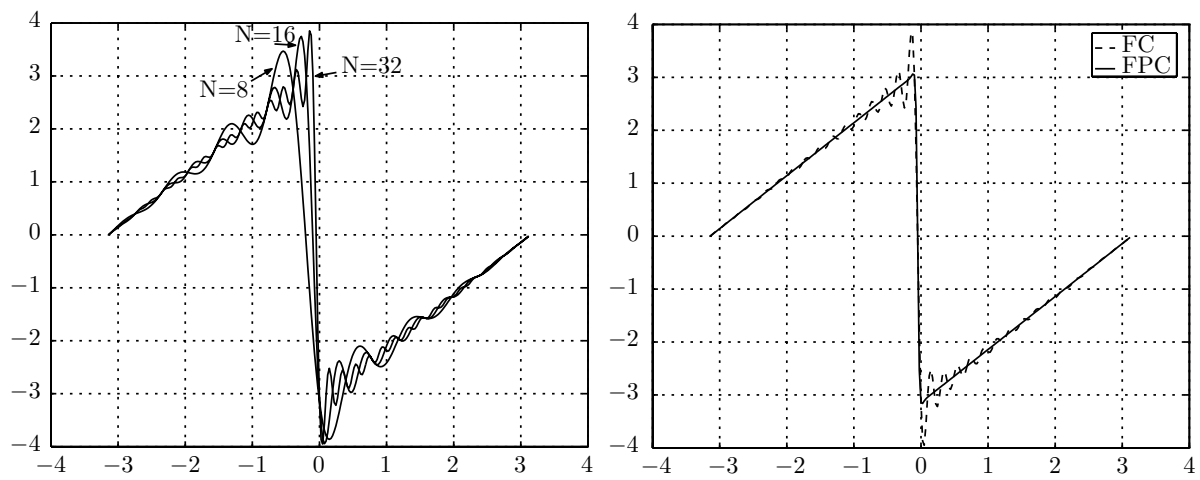

Figure 3.1. Gibbs oscillations by Fourier collocation (FC) approximation (left) and Fourier-Padé collocation (FPC) reconstruction (right) for the sawtooth function.

Thus we can simplify the Fourier-Padé collocation expansion as follows:

$$
R_{K, M}^{c}(x)=\sum_{j=0}^{2 N-1} u\left(x_{j}\right) w_{j}(x),
$$

where

$$
w_{j}(x)=\frac{Q_{M}^{c}\left(x_{j}\right)}{Q_{M}^{c}(x)}\left(\frac{1}{2 N} \sum_{k=-K}^{K-1} e^{i k\left(x-x_{j}\right)}\right) .
$$

Clearly, since $K=N-2 M$, for $M \neq 0$,

$$
w_{j}\left(x_{i}\right)=\left\{\begin{array}{c}
\frac{(N-2 M)}{N} \text { for } x_{i}=x_{j}, \\
\text { not always zero for } x_{i} \neq x_{j} .
\end{array}\right.
$$

Thus, $w_{j}(x)$ is not a Lagrange interpolation polynomial with the grids $x_{j}(0 \leq j \leq$ $2 N-1)$ defined here.
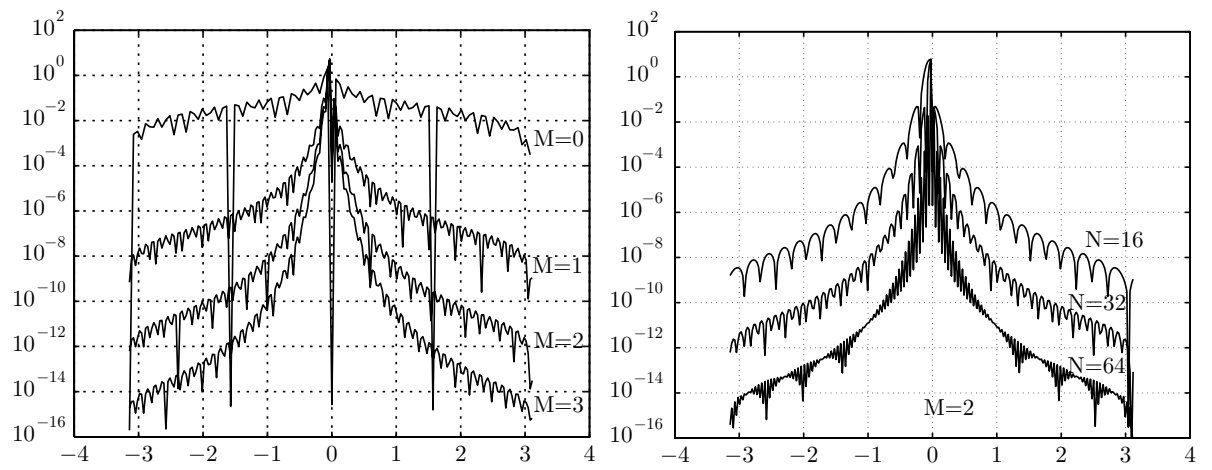

Figure 3.2. Convergence of the pointwise errors after the Fourier-Padé collocation reconstruction for the sawtooth function with a fixed $N$ (left) and a fixed $M$ (right), respectively. 
Example 2. We present the numerical results of the Fourier-Padé collocation method applied to the same example as defined in (2.12). As expected, the behavior of the Fourier-Padé collocation approach is similar to the one for the Fourier-PadéGalerkin approach as shown in Figures 3.1 and 3.2 .

\section{Applications}

In this section, we apply the Fourier-Padé collocation postprocessing procedure to the nonlinear hyperbolic and elliptic partial differential equations for which an initially smooth solution will develop a shock and sharp gradient in time, respectively. We discuss our numerical study of the inviscid Burgers' equation and twodimensional incompressible inviscid Boussinesq convection flow. The algorithm for solving these two problems with periodical boundary condition is straightforward and presented in the literature. A detailed description of the basic building blocks of the algorithm is provided in each subsection.
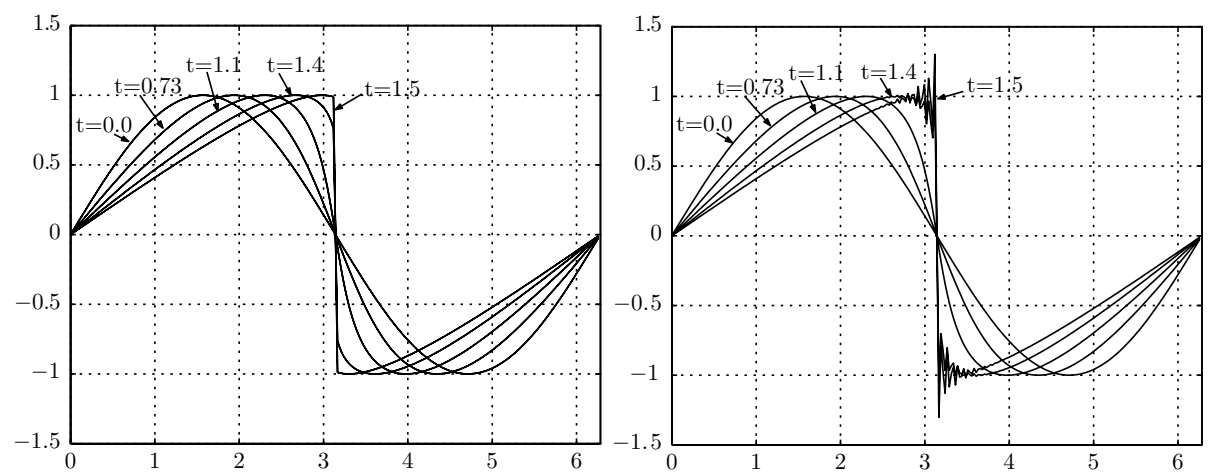

Figure 4.1. Evolutions of the exact solution (left) and the filtered (order=16) Fourier numerical solutions for the inviscid Burgers' equation at different times on grids 256 .
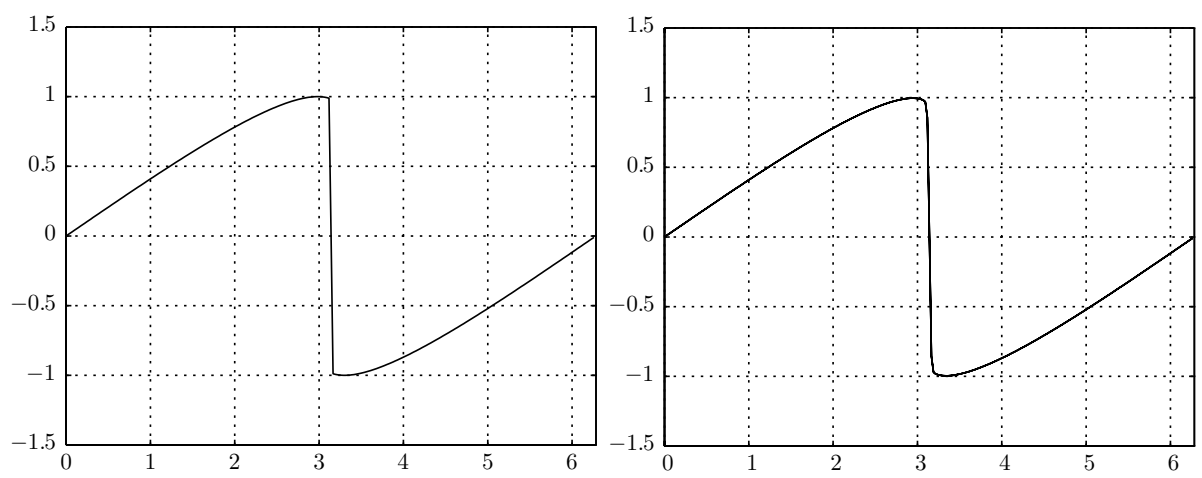

Figure 4.2. The exact solution (left) and after the Fourier-Padé reconstruction (right) for the filtered Fourier numerical solutions at $t=1.5 . N_{c}=64$ on grids 256 . 
4.1. Burgers' equation. The Burgers' equation is a suitable model for testing computational algorithms for flows where steep gradients or shocks are anticipated because it allows exact solutions for many combinations of initial and boundary conditions. Here we consider the inviscid Burgers' equation in one dimension,

$$
\frac{\partial u}{\partial t}+\frac{\partial}{\partial x}\left(\frac{1}{2} u^{2}\right)=0,
$$

with the initial condition

$$
u(x, 0)=\sin (x) .
$$

Figure 4.1 shows the evolution of the exact solution for the Burgers' equation. With the sine wave as an initial condition, the solution forms a stationary shock due to the nonlinearity in (4.1). The numerical solutions performed by the standard Fourier collocation methods are shown in Figure 4.1 (right). The third-order Runge-Kutta method is used for time integration, and the high-frequency components are smoothed out at each time step by using the exponential filter of order 16 in order to stabilize the scheme [18. As predicted, one can observe the Gibbs oscillations near the shock at $t=1.5$, a natural consequence when one considers the Fourier method to approximate a discontinuous solution.

We note that one should not attempt to reconstruct the function using all the modes in the Fourier spectrum. Depending on the order of the filtering used for the stability of the numerical scheme, only the Fourier coefficients from the lower one-half to two-thirds of the Fourier spectrum are used in the postprocessing reconstruction step, since the upper portion of the Fourier spectrum does not contain highly accurate information. Thus, an additional parameter for the cut-off frequency $N_{c}$ should be specified to determine the upper bound of the Fourier mode, information that will not contribute in the reconstruction step.

The numerical results in Figure 4.2 show that the oscillations have been mitigated after the Fourier-Padé reconstruction for the filtered Fourier numerical solutions at $t=1.5$. The pointwise errors shown in Figures 4.34 .4 demonstrate the successful
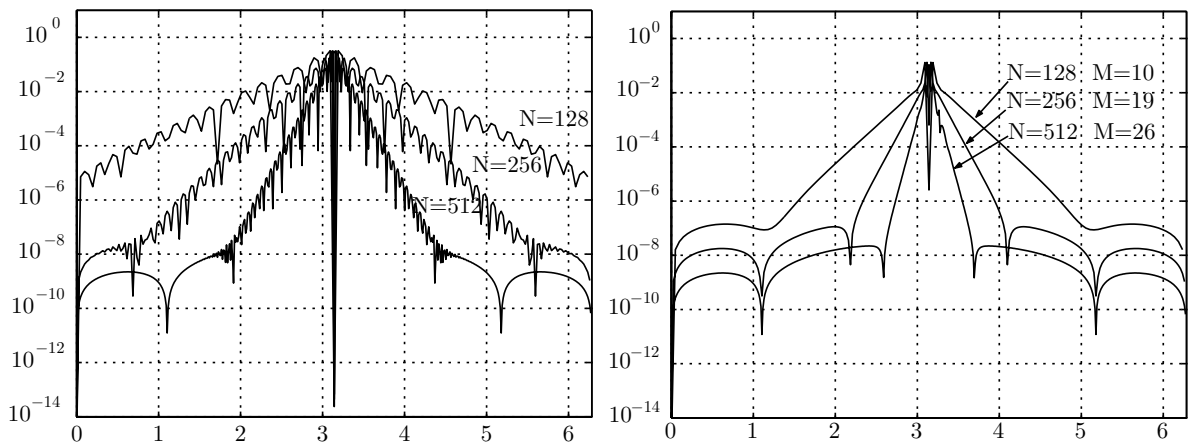

FiguRe 4.3. Convergence of the pointwise errors for filtered (order=16) Fourier collocation methods (left) and after the reconstruction by the Fourier-Padé collocation method for the inviscid Burgers' equation at $t=1.5$. 

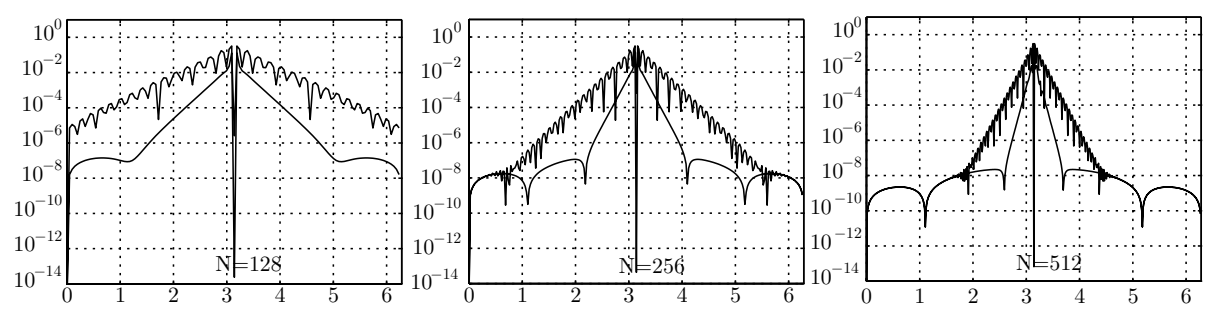

Figure 4.4. Comparison of the pointwise errors for filtered (order=16) Fourier methods and Fourier-Padé reconstruction for the inviscid Burgers' equation at $t=1.5$ on different size of the grids $128,256,512$ and $m=10,19,26$, respectively.

reduction of the spurious oscillations away from the discontinuity after the FourierPadé postprocessing. The exponential decays in errors are observed as $N$ and $M$ increase. Here we use the cut-off frequency $N_{c}=\frac{N}{2}$.

In this section, we present some preliminary results for the use of the FourierPadé reconstruction to recover the essentially nonoscillatory solution applied to the standard Fourier spectral simulations for the two-dimensional incompressible Boussinesq convection flow.

The mass conservation and the motion for an incompressible inviscid fluid with gravity as the only force acting on the fluid in the upward vertical direction has the form

$$
\begin{aligned}
\frac{\partial \rho}{\partial t}+\mathbf{u} \cdot \nabla \rho & =0 \\
\frac{\partial \mathbf{u}}{\partial t}+\mathbf{u} \cdot \nabla \mathbf{u}+\nabla p & =\left(\begin{array}{c}
0 \\
\rho g
\end{array}\right), \\
\nabla \cdot \mathbf{u} & =0,
\end{aligned}
$$

where $\rho$ is the density (or temperature), $\mathbf{u}=(u, v)$ is the velocity vector, $p$ is the pressure, and $g$ is the gravitational constant (normalized to 1 ).

For the two-dimensional flow of an incompressible fluid in the $x-y$ plane, mass conservation is satisfied by writing

$$
u=\frac{\partial \psi}{\partial y}, \quad v=-\frac{\partial \psi}{\partial x},
$$

where the scalar function $\psi(x, y, t)$ is the stream function. Let $\omega$ be the vorticity defined by $\omega=\nabla \times \mathbf{u}$. Then the above system can be rewritten as follows, the so-called stream function-vorticity formulation:

$$
\begin{aligned}
\frac{\partial \rho}{\partial t}+\mathbf{u} \cdot \nabla \rho & =0 \\
\frac{\partial \omega}{\partial t}+\mathbf{u} \cdot \nabla \omega & =-\frac{\partial \rho}{\partial x} \\
-\Delta \psi & =\omega .
\end{aligned}
$$

The initial condition is the same as the one in [15], consisting of a smooth bubble density in a zero flow field. The density function as an initial data is given as

$$
\rho(x, y, 0)=50 \rho_{1} \rho_{2}\left(1-\rho_{1}\right),
$$


where

$$
\rho_{1}= \begin{cases}\exp \left(1-\frac{\pi^{2}}{\pi^{2}-R_{1}^{2}}\right) & R_{1}^{2}=x^{2}+(y-\pi)^{2}<\pi^{2} \\ 0 & \text { otherwise }\end{cases}
$$

and

$$
\rho_{2}= \begin{cases}\exp \left(1-\frac{R_{3}^{2}}{R_{3}^{2}-R_{2}^{2}}\right) & R_{2}=|x-2 \pi|<R_{3}=1.95 \pi \\ 0 & \text { otherwise }\end{cases}
$$

The density contour at $t=0$ is shown in Figure 4.5, computed with $512^{2}$ grids. For this smooth initial data, the solution remains smooth for a short time, as shown in Figure 4.7. As the bubble rises to the right, however, the front of the bubble steepens into a sharp gradient. A large amount of vorticity is generated from the source term in the vorticity equation and deposited around the sharp density edge. Following 3 and [15, one can claim that if a solution develops a singularity in finite time, then the density gradient should grow exponentially.
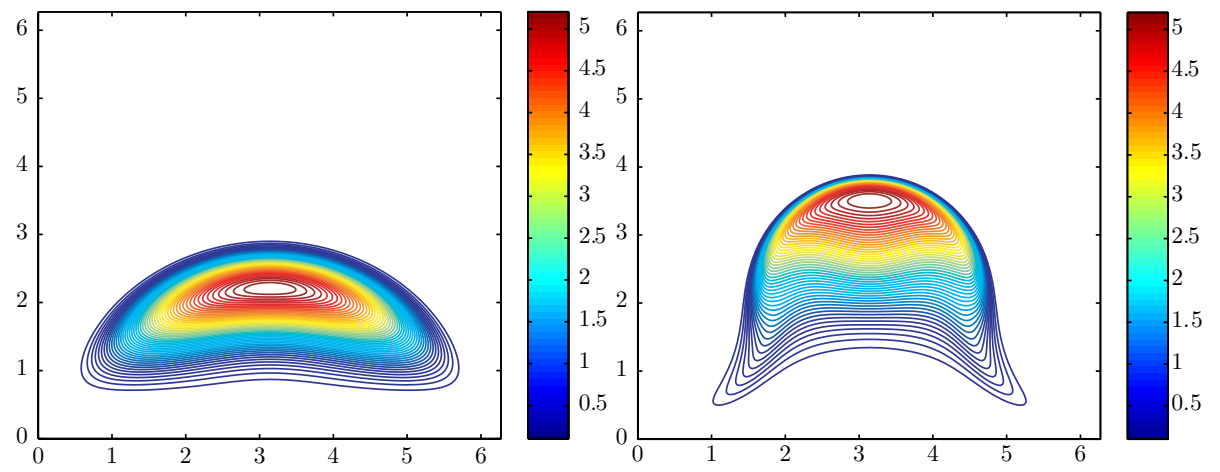

Figure 4.5. Density contour of the Boussinesq equations at $t=$ 0.0 (left) and $t=1.1$ (right) with the Fourier collocation methods on grids $512^{2}$.
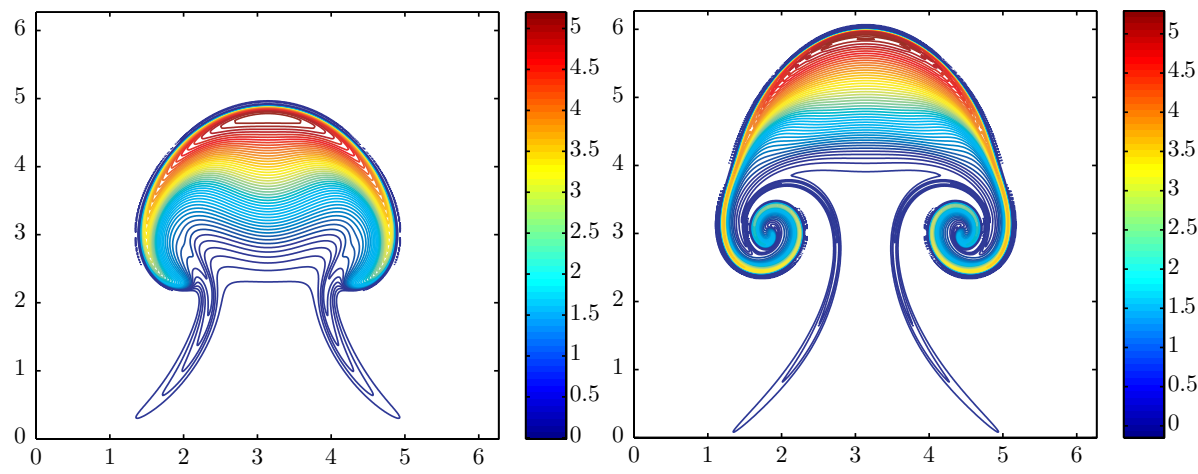

Figure 4.6. Density contour of the Boussinesq equations at $t=$ 2.2 (left) and $t=3.0$ (right) with the Fourier collocation methods on grids $512^{2}$. 


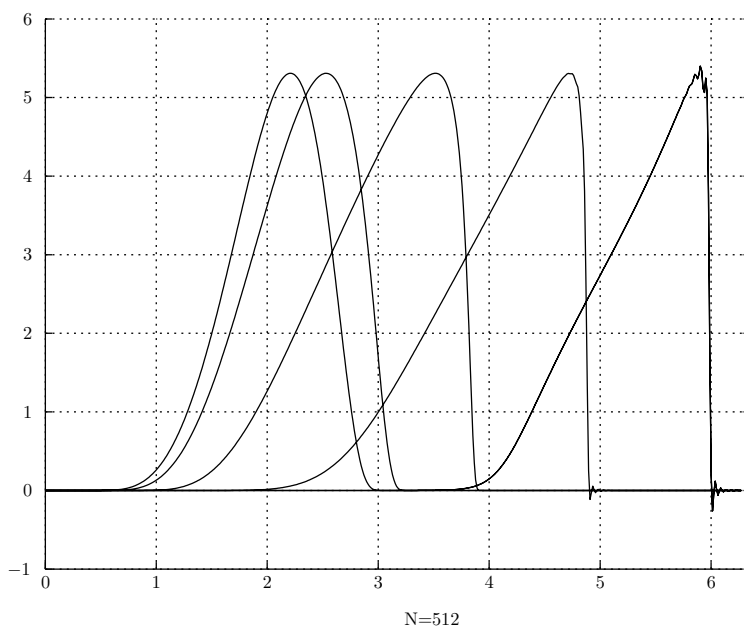

Figure 4.7. Evolution of density along the symmetry axis $x=\pi$ by the filtered (order $=16$ ) Fourier method on grids $512^{2}$.

To resolve the singularity, one would have to refine the grid continuously around the gradient front, a procedure that is neither realistic nor practical. Hence, it remains controversial whether there exists a finite time singularity in such flows. Various computational computational techniques have been developed [14, 15, [17, 19. Moving mesh computations have been performed in [19, which uses an adaptive grid strategy developed in [17. In [15, the ENO method has been applied for simulating the evolution of the bubble with the same initial setup we present here. Their numerical results are consistent with the experimental results in [1, demonstrating severe deformation of the bubble in the lower part while retaining the shape of the bubble on the top.

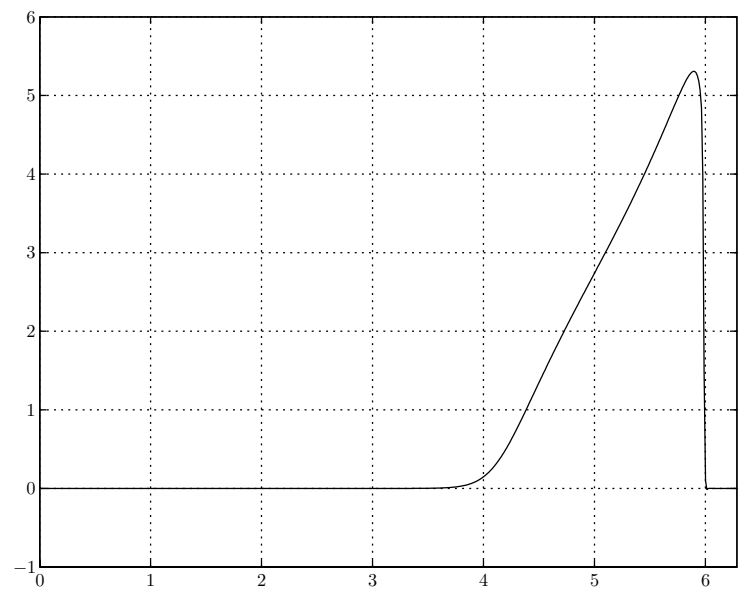

FiguRE 4.8. Density along the symmetry axis $x=\pi$ after reconstruction with the Fourier-Padé collocation method on grids $512^{2}$, $N_{c}=137, M=46$. 

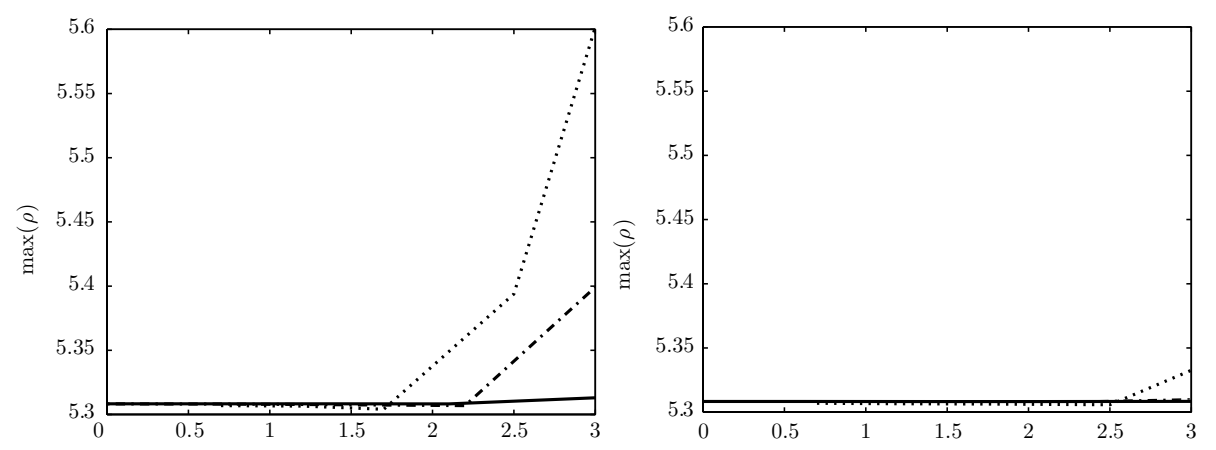

Figure 4.9. Time history of the maximum density along the symmetry axis $y=\pi$ : filtered Fourier (left) and after the Fourier-Padé reconstructions (right) with dots on grids $256^{2}, M=64, N_{c}=14$, dash-dot on grids $512^{2}, M=24, N_{c}=24$, and solid line on grids $1024^{2}, M=34, N_{c}=34$.
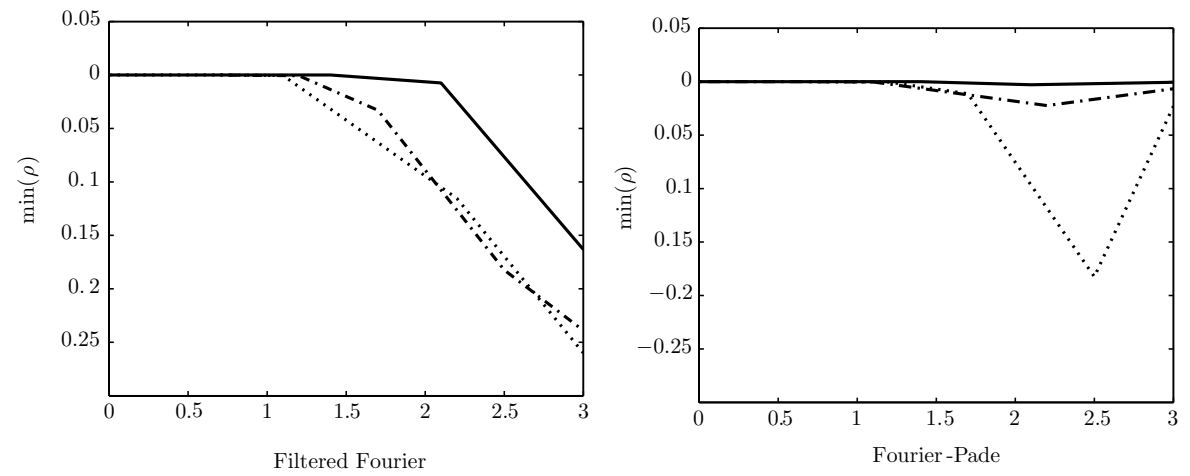

Figure 4.10. Time history of the minimum density along the symmetry axis $y=\pi$ : filtered Fourier (left) and after the FourierPadé reconstructions (right) with dots on grids $256^{2}, M=64$, $N_{c}=14$, dash-dot on grids $512^{2}, M=24, N_{c}=24$, and solid line on grids $1024^{2}, M=34, N_{c}=34$.

In our work, we use the standard Fourier collocation method in space, with a periodic boundary condition. The third-order TVD Runge-Kutta scheme is used for evolution in time for the equations of mass conservation and vorticity production. The exponential filters are applied at each time step in order to maintain the stability of the scheme, following the robust method in [18. The order of filter varies from 12 to 16 as the resolution of the simulation increases. The traditional Fourier collocation method results in disastrous oscillations when the solution develops a large gradient and the number of Fourier modes are not sufficient enough to fully resolve the gradient. Here we investigate a way of suppressing the spurious oscillations near singularity and an accurate recovery of the underlying nonoscillatory solution by using Fourier-Padé reconstruction as postprocessing as the final time step. As with the Burgers' equation, this approach is possible because the Fourier spectral simulation contains highly accurate information about the solution. 

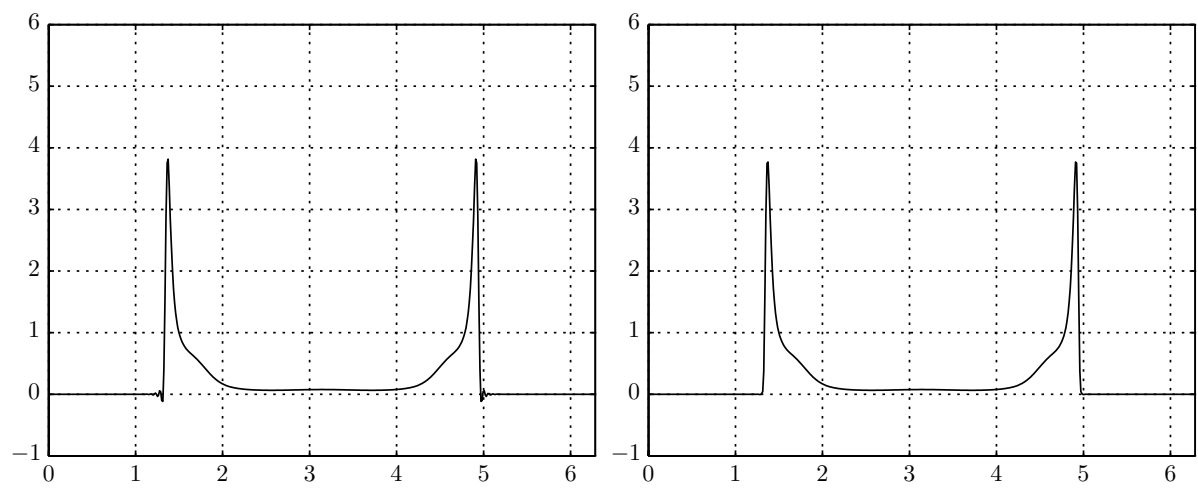

Figure 4.11. Density along the symmetry axis $y=3.91$ at $t=$ 3.0 with filtered Fourier collocation method (left) and after the Fourier-Padé reconstruction (right) on grids $512^{2}, M=26$, and $N_{c}=136$.
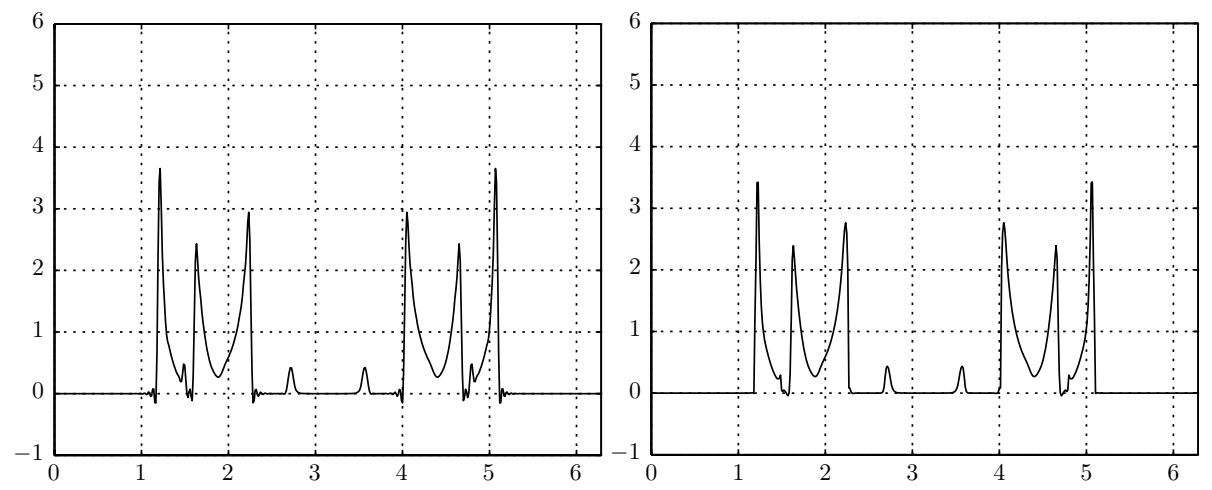

Figure 4.12. Density along the symmetry axis $x=\pi$ at $t=3.0$ by filtered Fourier collocation method (left) and after the FourierPadé reconstruction (right) on grids $512^{2}, M=49$, and $N_{c}=148$.

The Poisson equation (4.8) in the spectral discretization is expressed by $A \psi+$ $\psi B=\omega$ in a matrix equation. In order to solve this linear system and compute a vector $\psi$ on the discretization for a given vorticity $\omega$, the matrices $A$ and $B$ are cast in the lower and upper Schur form, and the transformed system is solved by backward substitution as in [2].

Figures 4.5 4.6 show the density profiles at different times, computed by standard filtered Fourier spectral simulation. As shown in [15], the sharp front density is developed from a smooth initial profile. Nonphysical oscillations start to appear when the numerical solution nearly reaches singularity in time if the numerical solution is underresolved. The one-dimensional cuts of the density at $y=\pi$ at different times are shown in Figure 4.7. As a result of the overshoot and undershoot of the Gibbs, the minimum $(=0.0)$ and the maximum $(=5.3084)$ value of the density are poorly represented in Figure 4.7 . 
One can effectively recover the nonoscillatory solution by mitigating the Gibbs oscillations with the use of the Fourier-Padé reconstruction as a postprocessing step in the numerical solution with the filtered Fourier collocation method.

For the Fourier-Padé reconstruction procedure, the Fourier modes from the top spectrum are discarded, as was done for the Burgers' equation, because the lower spectrum of the Fourier modes are accurately resolved and the numerical errors are usually associated with the high modes. Thus, to get the best reconstruction, one must select a reasonable cut-off frequency $N_{c}$.

In Figures 4.74.12, the numerical results show the time history of the minimum and maximum values of the density before and after the Fourier-Padé reconstruction procedure. One can observe that, for all practical purposes, the Gibbs overshoot and undershoot are effectively removed, even for the cases of the small scale structure shown in Figures 4.11 and 4.12

\section{Conclusion}

Using rational approximation based on the Fourier series, we have defined the explicit representations of the Fourier-Padé-Galerkin approximation and the FourierPadé collocation approximation in terms of the given continuous and discrete Fourier coefficients, respectively.

Numerical results for the Fourier-Padé-Galerkin and Fourier-Padé collocation methods show the successful mitigation of the Gibbs oscillations away from the singularity with spectral convergence, as the grids are refined and the degree of the denominator is increased.

Numerical simulations for the inviscid Burgers' equation and two-dimensional incompressible Boussinesq convection flow show the successful reduction of the Gibbs oscillations in the standard Fourier spectral simulations after postprocessing with Fourier-Padé reconstruction.

\section{ACKNOWLEDGMENTS}

We thank David Gottlieb for support of this project and for his useful suggestions. The conversation with Jan S. Hesthaven and Laura Lurati has been helpful.

\section{REFERENCES}

[1] G. K. BAtChelor, The stability of a large gas bubble moving through a liquid, J. Fluid Mech. 184 (1987) 399.

[2] R. H. Bartels and G. W. Stewart, Algorithm 432, solution of the matrix equation Ax + $x B=C$, Comm. ACM 15 (1972) 820-826.

[3] J. T. Beale, T. Kato, And A. Majda, Remarks on the breakdown of smooth solutions for the 3-D Euler equations, Comm. Math. Phys. 94 (1984) 61-66. MR0763762 (85j:35154)

[4] H. Cabannes, Padé Approximants Method and Its Applications to Mechanics, Lecture Notes in Physics, edited by J. Ehlers, K. Hepp, H. A. Weidenmüller, and J. Zittartz, Springer-Verlag, New York, 1976. MR0494843 (58:13627)

[5] A. Dold And B. Eckmann, Pade Approximation and Its Application, Springer-Verlag, New York, 1979.

[6] L. Emmel, S.M. Kaber, And Y. Maday, Padé-Jacobi filtering for spectral approximations of discontinuous solutions, Numer. Algorithms 33 (2003), no. 1-4, 251-264. MR2005567 (2004k:65233)

[7] T. A. Driscoll and B. Fornberg, A Padé-based algorithm for overcoming the Gibbs phenomenon, Numer. Algorithms 26 (2001), no. 1, 77-92. MR1827318 (2002b:65007)

[8] J. F. Geer, Rational trigonometric approximations using Fourier series partial sums, J. Sci. Comp. 10, no. 3 (1995) 325-356. MR1361091 (96k:42003) 
[9] J. GibBs, Fourier's series, Nature 59 (1898) 200.

[10] D. Gottlieb, M. Y. Hussaini, And S. A. Orszag, Introduction: Theory and applications of spectral methods, in Spectral Methods for Partial Differential Equations, edited by R. Voigt, D. Gottlieb, and M. Y. Hussaini, SIAM, Philadelphia, 1984, pp. 1-54. MR0758261 (86h:65142)

[11] D. Gottlieb and S. A. Orszag, Numerical Analysis of Spectral Methods: Theory and Application, CMBS-NSF Regional Conference Series in Applied Mathematics 26, SIAM, Philadelphia, 1977. MR0520152 (58:24983)

[12] D. Gottlieb AND C. W. Shu, The Gibbs phenomenon and its resolution, SIAM Review 39 (1997) 644-668. MR 1491051 (98m:42002)

[13] H. PADÉ, Mémoire sur les développements en fractions continues de la fonction exponentielle puvant servir d'introduction à la théorie des fractions continues algébriques, Ann. Fac. Sci. de l'Ec. Norm. Sup. 16 (1899) 395-436.

[14] A. Pumir And E. D. Siggia, Development of singular solutions to the axisymmetric Euler equations, Phys. Fluids A 4 (1992) 1472-1491. MR.1167779 (93c:76014)

[15] Weinan E and C. W. Shu, Small-scale structures in Boussinesq convection, Phys. Fluids 6, no. 1 (1994) 49-58. MR1252833 (94i:76075)

[16] E. B. Saff And R. S. Vargra, Pade and Rational Approximation: Theory and Application, Academic Press, New York, 1977. MR 0458010 (56:16213)

[17] H. Z. TANG AND T. TANG, Adaptive mesh methods for one- and two-dimensional hyperbolic conservation laws, SIAM J. Numer. Anal. 41 (2003), 487-515. MR.2004185 (2004f:65143)

[18] H. VAndeven, Family of spectral filters for discontinuous problems, J. Sci. Compt. 6 (1991) 159-192. MR1140344 (92k:65006)

[19] Z. R. Zhang, Moving Mesh Methods for Convection-dominated Equations and Nonlinear Conservation Laws, Ph.D. thesis, Hong Kong Baptist University, 2003.

Division of Applied Mathematics, Brown University, Providence, Rhode Island

E-mail address: msmin@cfm.brown.edu

Laboratoire Jacques-Louis Lions, Université Paris Vi, France

E-mail address: kaber@ann.jussieu.fr

Division of Applied Mathematics, Brown University, Providence, Rhode Island

E-mail address: wsdon@cfm.brown.edu 